VOL. 38 (1988) [365-371]

\title{
A DISCRETE CALCULUS OF VARIATIONS ALGORITHM
}

\author{
H.H.TAN AND R.B.POTTS
}

\begin{abstract}
An algorithm which has been developed to solve the problem of determining an optimal path of the hand of a robot is applied to various classical problems in the calculus of variations.
\end{abstract}

\section{INTRODUCTION}

In a recent paper [6] an algorithm has been described which gives a general and efficient solution to the problem of determining an optimal path of the hand or end effector of a robot manipulator subject to a variety of geometric, kinematic and dynamic constraints. The algorithm is based on a discrete form of the Lagrange equations of motion for the manipulator $[1,3,4]$.

To test the algorithm it has been applied to several classical calculus of variations problems for which the solutions are known. It is the purpose of this paper to report the success of the algorithm when applied to these and to illustrate its potential use for a wide class of optimisation problems.

\section{Optical Reflection}

The optical problem of reflection of light from a surface will be used to introduce notation and the general features of the discrete algorithm. Figure 1 illustrates the situation. A ray of light from $A\left(a_{1}, a_{2}\right)$ to $B\left(b_{1}, b_{2}\right)$ in a uniform medium in the upper half of the $q_{1}, q_{2}$ plane is reflected at $R(r, 0)$ on the boundary $q_{2}=0$ of a denser medium. According to optical theory, the actual path of the light ray is that which minimises the total passage time from $A$ to $B$, and from this the reflection law straight line rays with angle of incidence equal to angle of reflection - can be readily deduced.

Suppose a possible path, in general allowed to be curved, is incident at point $R$ on the surface. Discretise the path by introducing $L+1$ knots, $k=0, \ldots, L$, on the segment $A R$ and a further $M$ knots, $k=L+1, \ldots, L+M$, on the segment $R B$. For each of the $L$ intervals on the segment $A R$, the passage time is assumed to be $\Delta t$, and for each of the $M$ intervals on the segment $R B, \Delta u$, so that the total passage time, which has to be minimised, is

$$
Z=L \Delta t+M \Delta u
$$

Received 4th February, 1988

Copyright Clearance Centre, Inc. Serial-fee code: 0004-9729/88 \$A2.00+0.00. 


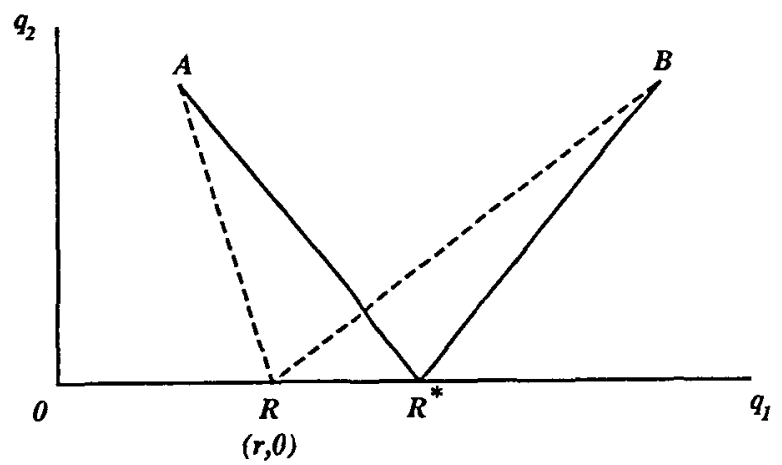

Figure 1: A ray of light from $A\left(a_{1}, a_{2}\right)$ to $B\left(b_{1}, b_{2}\right)$ in a uniform medium is reflected at $R$ on the boundary $q_{2}=0$. The optimal solution gives straight lines $A R^{*}$ and $R^{*} B$ with the angle of incidence equal to the angle of reflection. The dashed lines indicate the initial solution taken for the discrete algorithm.

Suppose the coordinates of the path at each knot $k$ are denoted by $q_{p}(k), k=$ $0, \ldots, L+M$, with $p=1,2$. The displacements are denoted by the forward differences

$$
\Delta q_{p}(k)=q_{p}(k+1)-q_{p}(k) \quad k=0, \ldots, L+M-1 .
$$

Basic to the discrete path planner algorithm is the use of the approximate trapezoidal or smoothing formulae used extensively by Greenspan [2] for $k=1, \ldots, L-1$

$$
\begin{aligned}
\dot{q}_{p}(k) & =-\dot{q}_{p}(k-1)+2[\Delta t]^{-1} \Delta q_{p}(k-1) \\
& =(-1)^{k} \dot{q}_{p}(0)-2(-1)^{k}[\Delta t]^{-1} \sum_{i=0}^{k-1}(-1)^{i} \Delta q_{p}(i),
\end{aligned}
$$

and for $k=L+1, \ldots, L+M$

$$
\begin{aligned}
\dot{q}_{p}(k) & =-\dot{q}_{p}(k-1)+2[\Delta u]^{-1} \Delta q_{p}(k-1) \\
& =(-1)^{k-L} \dot{q}_{p}(L)-2(-1)^{k}[\Delta u]^{-1} \sum_{i=L}^{k-1}(-1)^{i} \Delta q_{p}(i) .
\end{aligned}
$$

The speed of light throughout the uniform medium above the reflecting surface is constant so that

$$
\dot{q}_{1}(k)^{2}+\dot{q}_{2}(k)^{2}=C \quad k=0, \ldots, L+M, k \neq L
$$


The problem of determining the optimum ray path from $A$ to $B$ via a point on the reflecting surface is now expressed as a nonlinear program. The $7+2(L+M)$ program variables are $\Delta t, \Delta u, r, \dot{q}_{p}(0), \dot{q}_{p}(L), \Delta q_{p}(k)$ for $k=0, \ldots, L+M-1, p=1,2$. The initial and final points are given with $q_{p}(0)=a_{p}, q_{p}(L+M)=b_{p}$. The linear objective function to be minimised is given by $(2.1)$. The constraints to be satisfied are the linear relations

$$
\begin{gathered}
\sum_{k=0}^{L-1} \Delta q_{1}(k)=r-a_{1}, \\
\sum_{k=L}^{L+M-1} \Delta q_{1}(k)=b_{1}-r, \\
\sum_{k=0}^{L-1} \Delta q_{2}(k)=-a_{2}, \\
\sum_{k=L}^{L+M-1} \Delta q_{2}(k)=b_{2},
\end{gathered}
$$

and the $L+M+1$ nonlinear relations (2.5), the nonlinearity arising from the necessity to introduce (2.3) and (2.4).

The NLP can be solved using sequential linear programming (SLP), sequential quadratic programming (SQP) or the augmented Lagrangian method and in particular the NPSOL and MINOS packages [5] were used. These techniques require the Jacobian matrix formed by the partial derivatives of the nonlinear expressions, in this case only (2.5), with respect to the program variables. These derivatives are simple to evaluate. The NLP algorithms require an initial solution, if possible feasible, and a simple such choice is to assume straight line paths $A R$ and $R B$.

A particular test of the algorithm was run with the following data:

$$
C=1, a_{1}=-0.5, a_{2}=0.5, b_{1}=b_{2}=0.5 \text {. }
$$

From a cold start with $L=M=5$, the initial solution was chosen with $\Delta t=\Delta u=$ $0.1, r=-0.3$, and straight line segments from $A$ to $R$ and $R$ to $B$ with

$$
\Delta q_{1}(k)=\left(r-a_{1}\right) / L, \quad \Delta q_{2}(k)=-a_{2} / L, \quad k=0, \ldots, L-1
$$

and

$$
\Delta q_{1}(k)=\left(b_{1}-r\right) / M, \quad \Delta q_{2}(k)=b_{2} / M, \quad k=L, \ldots, L+M-1 .
$$

The initial values of the velocity variables were chosen to be

$$
\dot{q}_{1}(0)=0.3714, \dot{q}_{2}(0)=-0.9285, \dot{q}_{1}(L)=0.8480, \dot{q}_{2}(L)=0.5300 .
$$


With the use of the NPSOL package, the expected theoretical result of straight line paths $A R^{*}$ and $R^{*} B$ with $R^{*}=(0,0)$ was obtained with about 6 figure accuracy and with a CPU time of 10 seconds on a VAX-11/785 computer.

\section{Optical Refraction}

A similar test of the optimal path planner was performed for the problem of optical refraction. The reflection problem was modified by choosing below the boundary $q_{2}=0$ a uniform medium of different density and taking $B$ within this medium so that $b_{2}<0$. The modifications to the formulation are straightforward with the right hand side of the velocity constraints set to the appropriate values.

A particular test of the algorithm was run with

$$
a_{1}=-0.5, a_{2}=0.5, b_{1}=\sqrt{3} / 6, b_{2}=-0.5
$$

and with the refractive index of the medium above the interface $q_{2}=0$ equal to 1 and that below equal to $\sqrt{2}$. For a test run with $L=M=5$, the initial values taken were:

$$
\Delta t=\Delta u=0.1, r=-0.3, \Delta q_{1}(k)=\left(r-a_{1}\right) / L, \Delta q_{2}(k)=-a_{2} / L, k=0, \ldots, L-1
$$

and

$$
\Delta q_{1}(k)=\left(b_{1}-r\right) / M, \Delta q_{2}(k)=b_{2} / M, k=L, \ldots, L+M-1 .
$$

The initial values of the velocity variables were taken as :

$$
\dot{q}_{1}(0)=0.3714, \dot{q}_{2}(0)=-0.9285, \dot{q}_{1}(L)=0.5389, \dot{q}_{2}(L)=-0.4578
$$

With NPSOL, the expected result of straight line paths from $A$ to $R^{*}$ and $R^{*}$ to $B$ with $R^{*}=(0,0)$ was obtained with 6 figure accuracy and a CPU time of 17 seconds.

\section{Brachistochrone Problem}

As a third test of the algorithm, the classic brachistochrone problem was considered - determine the path $O B$ in the vertical $q_{1}, q_{2}$ plane (see Figure 2) giving minimum travel time for a frictionless particle starting from rest and acted upon by gravity. To be specific, if $B=(\pi,-2)$, the path is the cycloid

$$
q_{1}(t)=t-\sin t, \quad q_{2}(t)=-1+\cos t,
$$

with travel time equal to $\pi$. The effect of gravity forces the nonlinear constraint

$$
\dot{q}_{1}^{2}+\dot{q}_{2}^{2}+2 q_{2}=0
$$




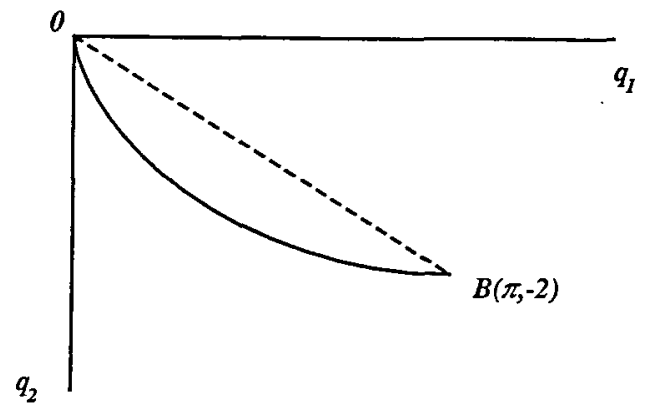

Figure 2: In the brachistochrone problem a frictionless particle falls under the action of gravity from $O$ at rest to a given point $B$. The dashed line is the straight path taken as the initial solution for the discrete algorithm; the curved path is the optimal solution, portion of a cycloid.

The discrete path planner takes the following form of an NLP with $2 L+1$ variables $\Delta t$ and $\Delta q_{p}(k)$ for $k=0, \ldots, L-1, p=1,2$ :

$$
\begin{aligned}
\text { minimise } \quad Z & =\Delta t, \\
\text { subject to } \quad \sum_{0}^{L-1} \Delta q_{1}(k) & =\pi, \\
\sum_{0}^{L-1} \Delta q_{2}(k) & =-2, \\
\dot{q}_{1}(k)^{2}+\dot{q}_{2}(k)^{2}+2 q_{2}(k) & =0, \quad k=1, \ldots, L .
\end{aligned}
$$

The values of $\dot{q}_{p}(k)$ are given by $(2.3)$, with $\dot{q}_{p}(0)=0$, and the values of $q_{2}(k)$ by

$$
q_{2}(k)=\sum_{i=0}^{k-1} \Delta q_{2}(i) \quad k=1, \ldots, L .
$$

Again a convenient initial solution for the NLP algorithm is the straight line path $O B$ with

$$
\Delta q_{1}(k)=\pi / L, \quad \Delta q_{2}(k)=-2 / L, \quad k=0, \ldots, L-1 .
$$

The result for $L=5$, obtained with a CPU time of 7.5 seconds, is shown in Figure 2. The travel time is 3.188 compared to the theoretical value $\pi$. For $L=10$, the graph of the solution, obtained with a CPU time of 26.9 seconds, is indistinguishable from the cycloid, and the travel time is now 3.153 . 


\section{Minimum Surface of Revolution Area}

As a final example consider the problem of determining the minimum surface of revolution area which in a simple case is formulated as the determination of the function $q_{2}=q_{2}\left(q_{1}\right)$ such that

$$
I=\int_{0}^{1} q_{2}\left(q_{1}\right)\left[1+q_{2}^{\prime}\left(q_{1}\right)^{2}\right]^{\frac{1}{2}} d q_{1}
$$

is a minimum subject to

$$
q_{2}(0)=1, \quad q_{2}(1)=\cosh 1
$$

The analytic solution is

$$
q_{2}^{*}=\cosh q_{1}, \quad I^{*}=\frac{1}{2}+\frac{1}{4} \sinh 2 .
$$

In the usual way, the path is discretised into $L$ intervals by taking $\Delta q_{1}=1 / L$. The $L+1$ variables for the nonlinear program are $\Delta q_{2}(k), k=0, \ldots, L-1$ and $q_{2}^{\prime}(0)$. The remaining derivatives are given by the smoothing formulae

$$
q_{2}^{\prime}(k+1)=-q_{2}^{\prime}(k)+2 L \Delta q_{2}(k), \quad k=0, \ldots, L-1 .
$$

The objective function to be minimised is taken as a discrete approximation to the integral $I$ given by (5.1). For example, the trapezoidal rule gives, apart from a factor $1 /(2 L)$

$$
Z=q_{2}(0)\left[1+q_{2}^{\prime}(0)^{2}\right]^{\frac{1}{2}}+2 \sum_{1}^{L-1} q_{2}(k)\left[1+q_{2}^{\prime}(k)^{2}\right]^{\frac{1}{2}}+q_{2}(L)\left[1+q_{2}^{\prime}(L)^{2}\right]^{\frac{1}{2}}
$$

The constraints are the linear relation

$$
\sum_{0}^{L-1} \Delta q_{2}(k)=-1+\cosh 1
$$

and the non-negativity relations

$$
q_{2}(k) \geqslant 0, \quad k=1, \ldots, L-1 .
$$

The initial solution was taken as

$$
\Delta q_{2}(k)=[-1+\cosh 1] / L, \quad k=0, \ldots, L-1, \quad q_{2}^{\prime}(0)=-1+\cosh 1 .
$$

For a test run using NPSOL and with $L=10$, about 3 figure accuracy was obtained with a CPU time of 7 seconds. For $L=20$ about 4 figure accuracy was obtained with a CPU time of 34 seconds. 


\section{DISCUSSION}

The numerical solution of problems in calculus of variations can be difficult because a potentially large function space has to be searched in order to find a good approximation to the function optimising the objective function. Practical problems in robotics involve functions of six variables corresponding to a robot's six degrees of freedom, and the 'curse of dimensions' has frustrated some attempts at solving optimal path planning. An algorithm which has been used successfully in robotics [6] has been applied in this paper to solve several classical calculus of variations problems. The algorithm has been proved to be applicable to general problems with various objectives and constraints.

\section{REFERENCES}

[1] L.Gotuso, 'On the energy theorem for the Lagrange equations in the discrete case', App. Math. Comput. 17 (1985), 120-136.

[2] D. Greenspan, Discrete Models (Addison-Wesley, Mass., U.S.A., 1973).

[3] C.P.Neuman and V.D.Tourassis, 'Discrete dynamic robot models', IEEE Trans. Systems Man Cybernet 15 (1985), 193-204.

[4] R.B.Potts, 'Discrete Lagrange equations', Bull. Austral. Math. Soc. 37 (1988), 227-233.

[5] Reports SOL 83-2OR and SOL 86-2 (Department of Operations Research, Stanford University, Stanford, U.S.A.).

[6] H.H.Tan and R.B.Potts, 'A minimum time discrete path planner', Univ. of Adel. App. Maths Res. Rep. UAAM-87-8 (December 1987).

Applied Mathematics Department

The University of Adelaide

G.P.O. Box 498

Adealide, S.A. 5001

Australia 